\title{
Evaluating the Representational Hub of Language and Vision Models
}

\author{
Ravi Shekhar ${ }^{\dagger}$, Ece Takmaz*, Raquel Fernández* and Raffaella Bernardi ${ }^{\dagger}$ \\ †University of Trento, ${ }^{*}$ University of Amsterdam \\ raffaella.bernardi@unitn.it raquel.fernandez@uva.nl
}

\begin{abstract}
The multimodal models used in the emerging field at the intersection of computational linguistics and computer vision implement the bottom-up processing of the "Hub and Spoke" architecture proposed in cognitive science to represent how the brain processes and combines multi-sensory inputs. In particular, the Hub is implemented as a neural network encoder. We investigate the effect on this encoder of various vision-and-language tasks proposed in the literature: visual question answering, visual reference resolution, and visually grounded dialogue. To measure the quality of the representations learned by the encoder, we use two kinds of analyses. First, we evaluate the encoder pre-trained on the different vision-and-language tasks on an existing diagnostic task designed to assess multimodal semantic understanding. Second, we carry out a battery of analyses aimed at studying how the encoder merges and exploits the two modalities.
\end{abstract}

\section{Introduction}

In recent years, a lot of progress has been made within the emerging field at the intersection of computational linguistics and computer vision thanks to the use of deep neural networks. The most common strategy to move the field forward has been to propose different multimodal tasks-such as visual question answering (Antol et al., 2015), visual question generation (Mostafazadeh et al., 2016), visual reference resolution (Kazemzadeh et al., 2014), and visual dialogue (Das et al., 2017)—and to develop task-specific models.

The benchmarks developed so far have put forward complex and distinct neural architectures, but in general they all share a common backbone consisting of an encoder which learns to merge the two types of representation to perform a certain task. This resembles the bottom-up processing in the 'Hub and Spoke' model proposed in Cognitive Science to represent how the brain processes and combines multi-sensory inputs (Patterson and Ralph, 2015). In this model, a 'hub' module merges the input processed by the sensor-specific 'spokes' into a joint representation. We focus our attention on the encoder implementing the 'hub' in artificial multimodal systems, with the goal of assessing its ability to compute multimodal representations that are useful beyond specific tasks.

While current visually grounded models perform remarkably well on the task they have been trained for, it is unclear whether they are able to learn representations that truly merge the two modalities and whether the skill they have acquired is stable enough to be transferred to other tasks. In this paper, we investigate these questions in detail. To do so, we evaluate an encoder trained on different multimodal tasks on an existing diagnostic task-FOIL (Shekhar et al., 2017)—designed to assess multimodal semantic understanding and carry out an in-depth analysis to study how the encoder merges and exploits the two modalities. We also exploit two techniques to investigate the structure of the learned semantic spaces: Representation Similarity Analysis (RSA) (Kriegeskorte et al., 2008) and Nearest Neighbour overlap $(\mathrm{NN})$. We use RSA to compare the outcome of the various encoders given the same vision-and-language input and NN to compare the multimodal space produced by an encoder with the ones built with the input visual and language embeddings, respectively, which allows us to measure the relative weight an encoder gives to the two modalities. 
In particular, we consider three visually grounded tasks: visual question answering (VQA) (Antol et al., 2015), where the encoder is trained to answer a question about an image; visual resolution of referring expressions (ReferIt) (Kazemzadeh et al., 2014), where the model has to pick up the referent object of a description in an image; and GuessWhat (de Vries et al., 2017), where the model has to identify the object in an image that is the target of a goal-oriented question-answer dialogue. We make sure the datasets used in the pre-training phase are as similar as possible in terms of size and image complexity, and use the same model architecture for the three pre-training tasks. This guarantees fair comparisons and the reliability of the results we obtain. ${ }^{1}$

We show that the multimodal encoding skills learned by pre-training the model on GuessWhat and ReferIt are more stable and transferable than the ones learned through VQA. This is reflected in the lower number of epochs and the smaller training data size they need to reach their best performance on the FOIL task. We also observe that the semantic spaces learned by the encoders trained on the ReferIt and GuessWhat tasks are closer to each other than to the semantic space learned by the VQA encoder. Despite these asymmetries among tasks, we find that all encoders give more weight to the visual input than the linguistic one.

\section{Related Work}

Our work is part of a recent research trend that aims at analyzing, interpreting, and evaluating neural models by means of auxiliary tasks besides the task they have been trained for (Adi et al., 2017; Linzen et al., 2016; Alishahi et al., 2017; Zhang and Bowman, 2018; Conneau et al., 2018). Within language and vision research, the growing interest in having a better understanding of what neural models really learn has led to the creation of several diagnostic datasets (Johnson et al., 2017; Shekhar et al., 2017; Suhr et al., 2017).

Another research direction which is relevant to our work is transfer learning, a machine learning area that studies how the skills learned by a model trained on a particular task can be transferred to learn a new task better, faster, or with less data. Transfer learning has proved successful in computer vision (e.g. Razavian et al. (2014)) as well as in computational linguistics (e.g., Conneau et al. (2017)). However, little has been done in this respect for visually grounded natural language processing models.

In this work, we combine these different research lines and explore transfer learning techniques in the domain of language and vision tasks. In particular, we use the FOIL diagnostic dataset (Shekhar et al., 2017) and investigate to what extent skills learned through different multimodal tasks transfer.

While transfering the knowledge learned by a pre-trained model can be useful in principle, Conneau et al. (2018) found that randomly initialized models provide strong baselines that can even outperfom pre-trained classifiers (see also Wieting and Kiela (2019)). However, it has also been shown that these untrained, randomly initialized models can be more sensitive to the size of the training set than pretrained models are (Zhang and Bowman, 2018). We will investigate these issues in our experiments.

\section{Visually Grounded Tasks and Diagnostic Task}

We study three visually grounded tasks: visual question answering (VQA), visual resolution of referring expressions (ReferIt), and goal-oriented dialogue for visual target identification (GuessWhat). While ReferIt was originally formulated as an object detection task (Kazemzadeh et al., 2014), VQA (Antol et al., 2015) and GuessWhat (de Vries et al., 2017) were defined as classification tasks. Here we operationalize the three tasks as retrieval tasks, which makes comparability easier.

- VQA: Given an image and a natural language question about it, the model is trained to retrieve the correct natural language answer out of a list of possible answers.

\footnotetext{
${ }^{1}$ The datasets are available at https://foilunitn.github.io/.
} 


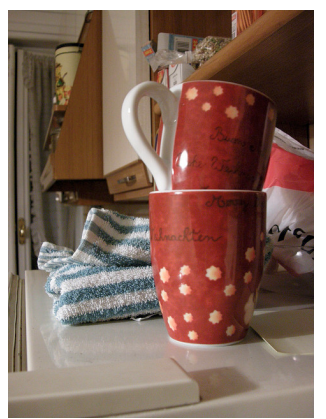

Q: How many cups are there?

A: Two.

ReferIt

The top mug.

\section{GuessWhat}

Q: Is it a mug?

A: Yes

$Q:$ Can you see the cup's handle?

A: Yes.

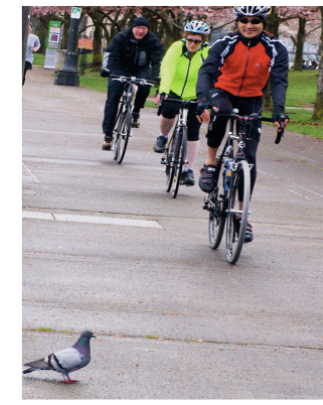

FOIL Diagnostic Task

original caption

Bikers approaching a bird.

foiled caption

Bikers approaching a dog.

Figure 1: Illustrations of the three visually-grounded tasks (left) and the diagnostic task (right).

- ReferIt: Given an image and a natural language description of an entity in the image, the model is asked to retrieve the bounding box of the corresponding entity out of a list of candidate bounding boxes.

- GuessWhat: Given an image and a natural language question-answer dialogue about a target entity in the image, the model is asked to retrieve the bounding box of the target among a list of candidate bounding boxes. The GuessWhat game also involves asking questions before guessing. Here we focus on the guessing task that takes place after the question generation step.

Figure 1 (left) exemplifies the similarities and differences among the three tasks. All three tasks require merging and encoding visual and linguistic input. In VQA, the system is trained to make a languagerelated prediction, while in ReferIt it is trained to make visual predictions. GuessWhat includes elements of both VQA and ReferIt, as well as specific properties: The system is trained to make a visual prediction (as in ReferIt) and it is exposed to questions (as in VQA); but in this case the linguistic input is a coherent sequence of visually grounded questions and answers that follow a goal-oriented strategy and that have been produced in an interactive setting.

To evaluate the multimodal representations learned by the encoders of the models trained on each of the three tasks above, we leverage the FOIL task (concretely, task 1 introduced by Shekhar et al. (2017)), a binary classification task designed to detect semantic incongruence in visually grounded language.

- FOIL (diagnostic task): Given an image and a natural language caption describing it, the model is asked to decide whether the caption faithfully describes the image or not, i.e., whether it contains a foiled word that is incompatible with the image (foil caption) or not (original caption). Figure 1 (right) shows an example in which the foiled word is "dog". Solving this task requires some degree of compositional alignment between modalities, which is key for fine-grained visually grounded semantics.

\section{Model Architecture and Training}

In cognitive science, the hub module of Patterson and Ralph (2015) receives representations processed by sensory-specific spokes and computes a multimodal representation out of them. All our models have a common core that resembles this architecture, while incorporating some task-specific components. This allows us to investigate the impact of specific tasks on the multimodal representations computed by the representational hub, which is implemented as an encoder. Figure 2 shows a diagram of the shared model components, which we explain in detail below.

\subsection{Shared components}

To facilitate the comparison of the representations learned via the different tasks we consider, we use pre-trained visual and linguistic features to process the input given to the encoders. This provides a 


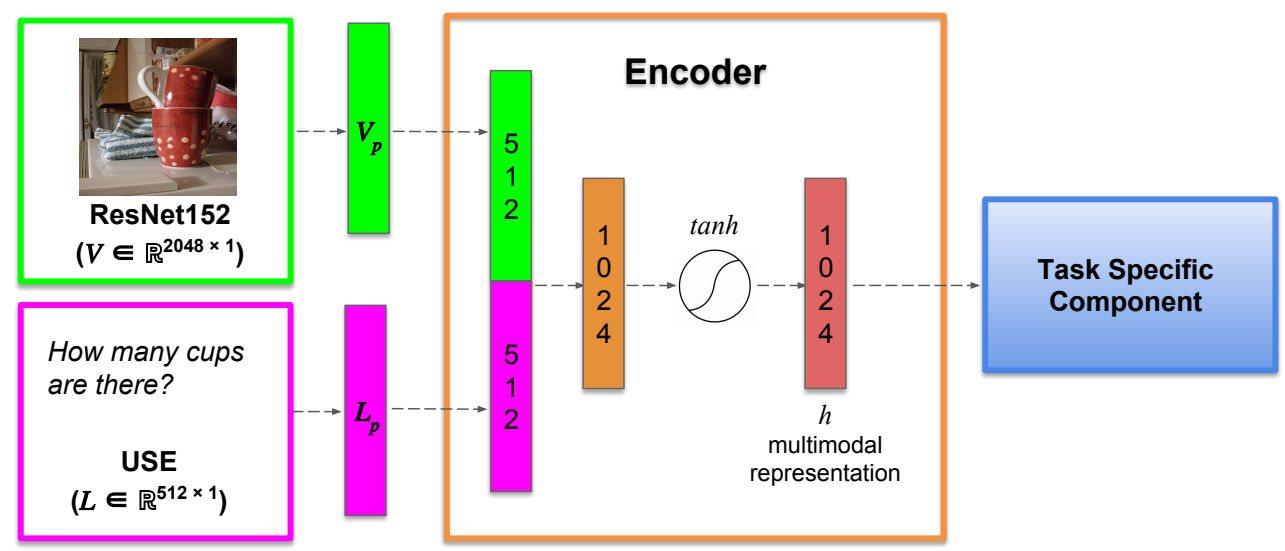

Figure 2: General model architecture, with an example from VQA as input. The encoder receives as input visual (ResNet152) and linguistic (USE) embeddings and merges them into a multimodal representation $(h)$. This is passed on to a task-specific component: an MLP in the case of the pre-training retrieval tasks and a fully connected layer in the case of the FOIL classification task.

common initial base across models and diminishes the effects of using different datasets for each specific task (the datasets are described in Section 5).

Visual and language embeddings To represent visual data, we use ResNet152 features (He et al., 2016), which yield state of the art performance in image classification tasks and can be computed efficiently. To represent linguistic data, we use Universal Sentence Encoder (USE) vectors (Cer et al., 2018) since they yield near state-of-the-art results on several NLP tasks and are suitable both for short texts (such as the descriptions in ReferIt) and longer ones (such as the dialogues in GuessWhat). ${ }^{2}$

In order to gain some insight into the semantic spaces that emerge from these visual and linguistic representations, we consider a sample of $5 \mathrm{~K}$ datapoints sharing the images across the three tasks and use average cosine similarity as a measure of space density. We find that the semantic space of the input images is denser ( 0.57 average cosine similarity) than the semantic space of the linguistic input across all tasks (average cosine similarity of 0.26 among VQA questions, 0.35 among ReferIt descriptions, and 0.49 among GuessWhat dialogues). However, when we consider the retrieval candidates rather than the input data, we find a different pattern: The linguistic semantic space of the candidate answers in VQA is much denser than the visual space of the candidate bounding boxes in ReferIt and GuessWhat $(0.93$ vs. 0.64 average cosine similarity, respectively). This suggests that the VQA task is harder, since the candidate answers are all highly similar.

Encoder As shown in Figure 2, ResNet152 visual features $\left(V \in \mathbb{R}^{2048 \times 1}\right)$ and USE linguistic features $\left(L \in \mathbb{R}^{512 \times 1}\right.$ ) are input in the model and passed through fully connected layers that project them onto spaces of the same dimensionality. The projected representations $\left(V_{p}\right.$ and $\left.L_{p}\right)$ are concatenated, passed through a linear layer, and then through a tanh activation function, which produces the final encoder representation $h$ :

$$
h=\tanh \left(W \cdot\left[V_{p} ; L_{p}\right]\right)
$$

where $W \in \mathbb{R}^{1024 \times 1024}, V_{p} \in \mathbb{R}^{512 \times 1}, L_{p} \in \mathbb{R}^{512 \times 1}$, and $[\cdot ; \cdot]$ represents concatenation.

\subsection{Task-specific components}

The architecture described above is shared by all the models we experiment with, which thus differ only with respect to their task-specific component.

\footnotetext{
${ }^{2}$ The dialogues in the GuessWhat?! dataset consist of 4.93 question-answer pairs on average (de Vries et al., 2017).
} 
Pre-training task component For the three tasks we consider, the final encoder representation $h$ is given to a Multi-Layer Perceptron (MLP), which generates either a language embedding (VQA model) or a visual embedding (ReferIt and GuessWhat models). The three task-specific models are trained with a cosine similarity loss, which aims to get the generated embedding closer to the ground truth embedding and farther away from any other embeddings in the list of candidates. Details of how, for each datapoint, the list of candidates is selected are provided in Section 5. The embeddings of such candidates are obtained with USE (for VQA) and ResNet (for ReferIt and GuessWhat). As mentioned above, the high density of the VQA candidate answers' space makes the task rather hard.

FOIL task component To evaluate the encoder representations learned by the pre-trained models, the task-specific MLPs are replaced by a fully connected layer, which is trained on the FOIL task using a cross-entropy loss. We train the FOIL task component using the following settings:

- $\mathbf{R a n d o m}_{2}$ The encoder weights are randomly initialized and the FOIL classifier layer is untrained. This provides a lower-bound baseline with random performance.

- Random The encoder weights are randomly initialized and then frozen while the FOIL classifier layer is trained on the FOIL task. This provides a strong baseline that is directly comparable to the task-specific setting explained next.

- Pre-trained (VQA, ReferIt, GuessWhat) The encoder weights are initialized with the Random setting's seeds and the model is trained on each of the tasks. The weights of the task-specific encoders are then frozen and the FOIL classifier layer is trained on the FOIL task. With this setting, we are able to diagnose the transfer and encoding properties of the pre-trained tasks.

- Fully trained on FOIL The encoder weights are initialized with the Radom setting's seeds. Then the full model is trained on the FOIL task, updating the weights of the projected vision and language layers, the encoder, and the FOIL layer. This provides the upper bound on the FOIL classification performance, as the entire model is optimized for this task from the start.

\section{Experimental Setup}

We provide details on the data sets and the implementation settings we use in our experiments.

Pre-training datasets For the three visually grounded tasks, we use the VQA.v1 dataset by Antol et al. (2015), the RefCOCO dataset by Yu et al. (2016), and the GuessWhat?! dataset by de Vries et al. (2017) as our starting point. All these datasets have been developed with images from MS-COCO (Lin et al., 2014). We construct common image datasets for by taking the intersection of the images in the three original datasets. This results in a total of 14,458 images. An image can be part of several data points, i.e, it can be paired with more than one linguistic input. Indeed, the 14,458 common images correspond to 43,374 questions for the VQA task, 104,227 descriptions for the ReferIt task, and 35,467 dialogues for the GuessWhat task.

To obtain datasets of equal size per task that are as similar as possible, we filter the resulting data points according to the following procedure:

1. For each image, we check how many linguistic items are present in the three datasets and fix the minimum number $(k)$ to be our target number of linguistic items paired with that image.

2. We select $n$ data points where the descriptions in ReferIt and dialogues in GuessWhat concern the same target object (with $n \leq k$ ).

3. Among the $n$ data points selected in the previous step, we select the $m$ data points in VQA where the question or the answer mention the same target object (computed by string matching).

4. We make sure all the images in each task-specific dataset are paired with exactly $k$ linguistic items; if not, we select additional ones randomly until this holds. 


\begin{tabular}{l|cc|ccc}
\hline & \multicolumn{2}{|c|}{ common image datasets } & \multicolumn{3}{c}{ FOIL dataset } \\
& training & validation & training & validation & testing \\
\hline \# images & 13,058 & 1,400 & 63,240 & 13,485 & 20,105 \\
\# language & 27,374 & 2,942 & 358,182 & 37,394 & 126,232 \\
\hline
\end{tabular}

Table 1: Statistics of the datasets used for the pre-training tasks and the FOIL task.

This results in a total of 30,316 data points per dataset: 14,458 images shared across datasets, paired with 30,313 linguistic items. We randomly divided this common image dataset into training and validation sets at the image level. The training set consists of 13,058 images (paired with 27,374 linguistic items) and the validation set of 1,400 images (paired with 2,942 linguistic items). Table 1 provides an overview of the datasets.

As mentioned in Section 3, we operationalize the three tasks as retrieval tasks where the goal is to retrieve the correct item out of a set of candidates. In the VQA.v1 dataset (multiple choice version), there are 18 candidate answers per question. In GuessWhat?! there are on average 18.71 candidate objects per dialogue, all of them appearing in the image. We take the same list of candidate objects per image for the ReferIt task.

FOIL dataset The FOIL dataset consists of image-caption pairs from MS-COCO and pairs where the caption has been modified by replacing a noun in the original caption with a foiled noun, such that the foiled caption is incongruent with the image - see Figure 1 for an example and Shekhar et al. (2017) for further details on the construction of the dataset. ${ }^{3}$ The dataset contains 521,808 captions $(358,182$ in training, 37,394 in validation and 126,232 in test set) and 96,830 images (63,240,13,485 and 20,105, in training, validation and test set, respectively) - see Table 1. All the images in the test set do not occur either in the FOIL training and validation set, nor in the common image dataset described above and used to pre-train the models.

Implementation details All models are trained using supervised learning with ground truth data. We use the same parameters for all models: batch size of 256 and Adam optimizer (Kingma and Ba, 2014) with learning rate 0.0001 . All the parameters are tuned on the validation set. Early stopping is used while training, i.e., training is stopped when there is no improvement on the validation loss for 10 consecutive epochs or a maximum of 100 epochs, and the best model is taken based on the validation loss.

\section{Results and Analysis}

We carry out two main blocks of analyses: one exploiting FOIL as diagnostic task and the other one investigating the structure of the semantic spaces produced by the pre-trained encoders when receiving the same multimodal inputs.

Before diving into the results of these analyses, we evaluate the three task-specific models on the tasks they have been trained for. Since these are retrieval tasks, we compute precision at rank1 (P@1) on the validation sets and compare the results to chance performance. Given the number of candidate answers and objects per task in our datasets, chance P@ 1 is 0.055 for VQA and 0.05 for ReferIt and GuessWhat. Our task-specific models obtain P@1 values of 0.14 for VQA (mean rank 2.84), 0.12 for ReferIt (mean rank 3.32), and 0.08 for GuessWhat (mean rank 4.14). Not surprisingly given the challenging nature of these tasks, the results are not high. Nevertheless, the representations learned by the models allow them to perform above chance level and thus provide a reasonable basis for further investigation.

\footnotetext{
${ }^{3}$ Madhysastha et al. (2018) found that an earlier version of the FOIL dataset was biased. We have used the latest version of the dataset available at https://foilunitn.github.io/, which does not have this problem.
} 


\subsection{Analysis via diagnostic task}

In this first analysis, we assess the quality of the multimodal representations learned by the three multimodal tasks considered in terms of their potential to perform the FOIL task, i.e., to spot semantic (in)congruence between an image and a caption. Besides comparing the models with respect to task accuracy, we also investigate how they learn to adapt to the FOIL task over training epochs, how much data they need to reach their best performance, and how confident they are about the decisions they make.

FOIL accuracy Table 2 shows accuracy results on the FOIL task for the different training settings described in Section 4.2. We report accuracy for the task overall, as well as accuracy on detecting original and foiled captions. As expected, the Random 2 setting yields chance performance ( $\approx 50 \%$ overall, with a surprisingly strong preference for classifying captions as foiled). The model fully trained on FOIL achieves an accuracy of $67.59 \%$. This confirms that the FOIL task is challenging, as shown by Shekhar et al. (2017), even for models that are optimized to solve it. The Random setting, where a randomly initialized encoder is trained on the FOIL task, yields $53.79 \%$ accuracy overall - higher than the chance lower bound by Random 2 , but well below the upper bound set by the fully trained model.

The key results of interest for our purposes in this paper are those achieved by the models where the encoder has been pre-trained on each of the three multimodal tasks we study. We observe that, like the Random encoder, the pre-trained encoders achieve results well below the upper bound. The VQA encoder yields results comparable to Random, while ReferIt and GuessWhat achieve slightly higher results: $54.02 \%$ and $54.18 \%$, respectively. This trend is much more noticeable when we zoom into the accuracy results on original vs. foiled captions. All models (except Random 2 ) achieve lower accuracy on the foil class than on the original class. However, the GuessWhat encoder performs substantially better than the rest: Its foil accuracy is not only well above the Random encoder, but also around $2 \%$ points over the fully trained model (49.34\% vs. $47.52 \%)$. The ReferIt encoder also performs reasonably well (on a par with the fully trained model), while the VQA encoder is closer to Random.

This suggests that the ReferIt and the GuessWhat encoders do learn a small degree of multimodal understanding skills that can transfer to new tasks. The VQA encoder, in contrast, seems to lack this ability by and large.

\begin{tabular}{l|c|cc}
\hline & overall & original & foiled \\
\hline Random $_{2}$ & 49.99 & 0.282 & 99.71 \\
Random & 53.79 & 65.33 & 42.25 \\
\hline VQA & 53.78 & 66.09 & 41.48 \\
ReferIt & 54.02 & 60.39 & 47.66 \\
GuessWhat & 54.18 & 59.02 & 49.34 \\
\hline Fully FOIL & 67.59 & 87.66 & 47.52 \\
\hline
\end{tabular}

Table 2: Accuracy on the FOIL task for the best model of each training setting.

Learning over time In order to better understand the effect of the representations learned by the pretrained encoders, we trace the evolution of the FOIL classification accuracy over time, i.e., over the first 50 training epochs. As shown in Figure 3a, all the pre-trained models start with higher accuracy than the Random model. This shows that the encoder is able to transfer knowledge from the pre-trained tasks to some extent. The Random model takes around 10 epochs to catch up and after that it does not manage to improve much. The evolution of the accuracy achieved by the ReferIt and GuessWhat encoders is relatively smooth, i.e., it increases progressively with further training epochs. The one by the VQA model, in contrast, is far less stable. 


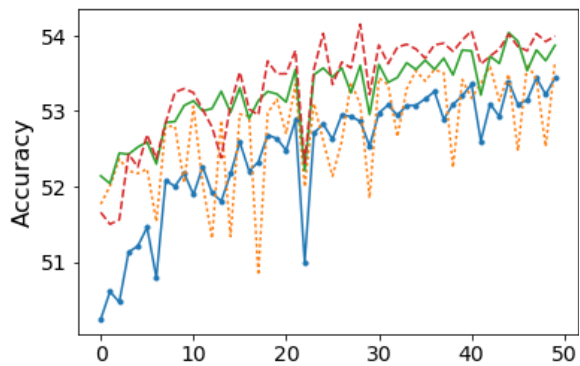

(a) Training epochs

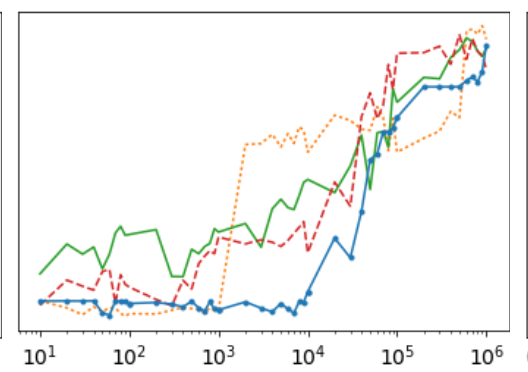

(b) Size of FOIL training set (log scaled).

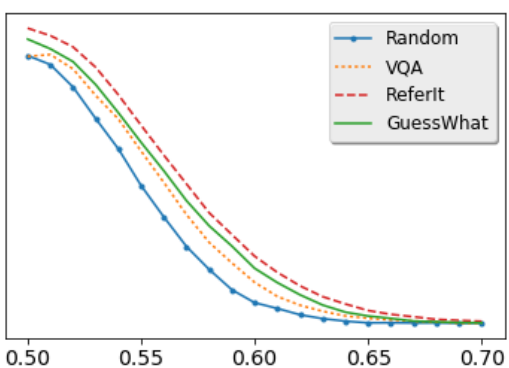

(c) AUC indicating confidence.

Figure 3: Comparisons among the pre-trained encoders and the randomly initialized encoder, regarding their accuracy over training epochs, with varying data size, and across different decision thresholds.

Size of FOIL training data Next, we evaluate how the accuracy achieved by the models changes when varying the size of the FOIL training set. By controlling the amount of training data, we can better tease apart whether the performance of the pre-trained models is due to the quality of the encoder representations or simply to the amount of training the models undergo on the FOIL task itself. Figure $3 \mathrm{~b}$ gives an overview. The GuessWhat encoder has a clear advantage when very little training data is available, while the other encoders start at chance level. Both GuessWhat and ReferIt increase their accuracy relatively smoothly as more data is provided, while for the VQA model there is a big jump in accuracy once enough FOIL data is available. Again, this suggests that the representations learned by the GuessWhat encoder are of somewhat higher quality, with more transferable potential.

Confidence Finally, we analyse the confidence of the models by measuring their Area Under the Curve (AUC). We gradually increase the classification threshold from 0.5 to 0.7 by an interval of 0.01 . This measures the confidence of the classifier in making a prediction. As shown in Figure 3c, all models have rather low confidence (when the threshold is 0.7 they are all at chance level). The Random model exhibits the lowest confidence, while the ReferIt model is slightly more confident in its decisions than the rest, followed by the GuessWhat model.

\subsection{Analysis of the multimodal semantic spaces learned by the encoders}

In this section, we analyse the encoders by comparing the similarity of the multimodal spaces they learn and by comparing the learned multimodal spaces to the visual and linguistic representations they receive as input in terms on nearest neighbours.

Representation similarity analysis Representation Similarity Analysis (RSA) is a technique from neuroscience (Kriegeskorte et al., 2008) that has been recently leveraged in computational linguistics, for example to compare the semantic spaces learned by artificial communicating agents (Bouchacourt and Baroni, 2018). It compares different semantic spaces by comparing their internal similarity relations, given a common set $N$ of input data points. Each input $k \in N$ is processed by an encoder for a given task $T i$, producing vector $h_{T i}^{k}$. Let $H_{T i}^{N}$ be the set of vector representations created by the encoder of $T i$ for all the items in $N$; and let $H_{T j}^{N}$ be the corresponding set of representations by the encoder of task $T j$. These two semantic spaces, $H_{T i}^{N}$ and $H_{T j}^{N}$, are not directly comparable as they have been produced independently. RSA remedies this by instead comparing their structure in terms of internal similarity relations. By computing cosine similarity between all pairs of vectors within each semantic space, we obtain a vector of cosine similarities per space, which captures its internal structure. These similarity vectors have identical dimensionality, namely $N(N-1) / 2$ ) values, and hence can be directly compared by computing Spearman correlation between them. The resulting RSA scores (corresponding to the aforementioned Spearman correlation coefficients) tell us the extent to which the two sets of representations are structurally similar. 


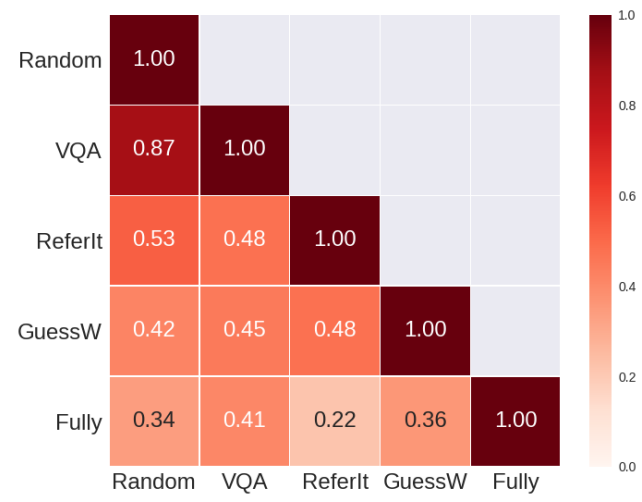

Figure 4: RSA scores indicating degree of structural similarity between the multimodal semantic spaces produced by the various encoders when receiving 5,000 data points from the FOIL test set consisting of unique images paired with their original captions.

The outputs of the encoders are compared when the same set of inputs is given. We give as input 5,000 data points from the FOIL test set, randomly sampled from only the ones with original captions and containing unique images, and compare the representations produced by the encoders under investigation. Figure 4 shows that the semantic space produced by the encoder fully trained on FOIL is rather different from all the other models, and that the VQA semantic space is very similar to the one produced by the randomly initialized encoder.

Nearest neighbour overlap We analyse the encoder representations using nearest neighbour overlap. Collell and Moens (2018) proposed this measure to compare the structure of functions that map concepts from an input to a target space. It is defined as the number of $k$ nearest neighbours that two paired vectors share in their respective semantic space. For instance, if $k=3$ and the 3 nearest neighbours of the vector for 'cat' $v_{\text {cat }}$ in space $V$ are $\left\{v_{\text {dog }}, v_{\text {tiger }}, v_{\text {lion }}\right\}$, and those of the vector of 'cat' $z_{\text {cat }}$ in space $Z$ are $\left\{v_{\text {mouse }}, v_{\text {tiger }}, v_{\text {lion }}\right\}$, the nearest neighbour overlap $(\mathrm{NN})$ is 2 . The value is then normalized with respect to the number of data points and the number of $k$ nearest neighbours.

\begin{tabular}{l|cc|cc}
\hline & \multicolumn{2}{|c|}{$k=1$} & \multicolumn{2}{c}{$k=10$} \\
& ResNet152 & USE & ResNet152 & USE \\
\hline Random & 0.829 & 0.363 & 0.876 & 0.365 \\
VQA & 0.638 & 0.350 & 0.703 & 0.386 \\
ReferIt & 0.754 & 0.346 & 0.780 & 0.366 \\
GuessWhat & 0.658 & 0.329 & 0.689 & 0.359 \\
Fully FOIL & 0.171 & 0.254 & 0.246 & 0.291 \\
\hline
\end{tabular}

Table 3: Average nearest neighbour overlap between the encoder multimodal representations and the ResNet152 and USE embeddings, respectively.

We take the encoder to be a mapping function from each of the modality-specific representations to the multimodal space, and we use the NN measure to investigate whether the structure of the multimodal space produced by the encoder is closer to the visual ResNet152 embeddings or to the linguistic USE embeddings given as input. We use simple visual and language inputs, namely, objects and the word corresponding to their object category. We consider the 80 object categories of MS-COCO (e.g., dog, car, etc.) and obtain their USE representations. We build their visual ResNet152 embedding by selecting 100 images for each category from MS-COCO, and then compute their average. We compute the NN by setting $k=1$ and $k=10$. The results, given in Table 3 , show that the multimodal spaces learned by all the models (except the model with the encoder fully trained on the FOIL task) are much closer to 
the visual input space than to the linguistic one. This behaviour could be related to the different density of the visual and linguistic semantic spaces of the input data we pointed out in Section 4.1, where we observed that input images have higher average cosine similarity than input questions, descriptions, and dialogues, respectively.

\section{Conclusion}

Our goal in this paper has been to evaluate the quality of the multimodal representations learned by an encoder - the core module of all the multimodal models used currently within the language and vision community - which resembles the cognitive representational hub described by Patterson and Ralph (2015). Furthermore, we investigated the transfer potential of the encoded skills, taking into account the amount of time (learning epochs) and training data the models need to adapt to a new task and with how much confidence they make their decisions. We studied three multimodal tasks, where the encoder is trained to answer a question about an image (VQA), pick up the object in an image referred to by a description (ReferIt), and identify the object in an image that is the target of a goal-oriented questionanswer dialogue (GuessWhat). To carry out this analysis, we have evaluated how the pre-trained models perform on a diagnostic task, FOIL (Shekhar et al., 2017), designed to check the model's ability to detect semantic incongruence in visually grounded language.

Overall, we found that none of the three tasks under investigation leads to learning fine-grained multimodal understanding skills that can solve the FOIL task, although there are differences among tasks. Our analysis shows that the VQA task is easier to learn (the model achieves a rather high mean rank precision). However the multimodal encoding skills it learns are less stable and transferable than the ones learned through the ReferIt and GuessWhat tasks. This can be seen by the large amount of data the model has to be exposed to in order to learn the FOIL classification task and by the unstable results over training epochs. None of the models transfers their encoding skills with high confidence, but again the VQA model does it to a lower extent.

The RSA analysis confirms the higher similarity of the multimodal spaces generated by the ReferIt and GuessWhat encoders and the high similarity between the VQA space and the space produced by the randomly initialized encoder. From the NN analysis, it appears that for all models (except for the one fully trained on the FOIL task) the visual modality has higher weight than the linguistic one in the construction of the multimodal representations.

These differences among tasks could be due to subtle parallelisms with the diagnostic task: ReferIt and Guess What may resemble some aspects of FOIL, since these three tasks revolve around objects (the foiled word is always a noun), while arguably the VQA task is more diverse as it contains questions about, e.g., actions, attributes, or scene configurations. In future work, it would be interesting to evaluate the models on different diagnostic datasets that prioritise skills other than object identification.

\section{Acknowledgements}

We kindly acknowledge the Leibniz-Zentrum für Informatik, Dagstuhl Seminar 19021 on Joint Processing of Language and Visual Data for Better Automated Understanding. The Amsterdam team was partially funded by the Netherlands Organisation for Scientific Research (NWO) under VIDI grant nr. 27689-008, Asymmetry in Conversation. We gratefully acknowledge the support of NVIDIA Corporation with the donation to the University of Trento of the GPUs used in our research.

\section{References}

Adi, Y., E. Kermany, Y. Belinkov, O. Lavi, and Y. Goldberg (2017). Fine-grained Analysis of Sentence Embeddings Using Auxiliary Prediction Tasks. In International Conference on Learning Representations (ICLR). 
Alishahi, A., M. Barking, and G. Chrupała (2017). Encoding of phonology in a recurrent neural model of grounded speech. In Proceedings of the 21st Conference on Computational Natural Language Learning (CoNLL 2017), pp. 368-378. Association for Computational Linguistics.

Antol, S., A. Agrawal, J. Lu, M. Mitchell, D. Batra, C. L. Zitnick, and D. Parikh (2015). VQA: Visual question answering. In International Conference on Computer Vision (ICCV).

Barrault, L., F. Bougares, L. Specia, C. Lala, D. Elliott, and S. Frank (2018). Findings of the third shared task on multimodal machine translation. In Proceedings of the Third Conference on Machine Translation (WMT), Volume 2, pp. 304323. Association for Computational Linguistics.

Bouchacourt, D. and M. Baroni (2018). How agents see things: On visual representations in an emergent language game. In Proceedings of the 2018 Conference on Empirical Methods in Natural Language Processing, pp. 981-985. Association for Computational Linguistics.

Cer, D., Y. Yang, S.-y. Kong, N. Hua, N. Limtiaco, R. S. John, N. Constant, M. Guajardo-Cespedes, S. Yuan, C. Tar, et al. (2018). Universal sentence encoder. arXiv preprint arXiv:1803.11175.

Collell, G. and M.-F. Moens (2018). Do neural network cross-modal mappings really bridge modalities? arXiv preprint arXiv:1805.07616.

Conneau, A., D. Kiela, H. Schwenk, L. Barrault, and A. Bordes (2017). Supervised learning of universal sentence representations from natural language inference data. In Proceedings of the 2017 Conference on Empirical Methods in Natural Language Processing, pp. 670-680.

Conneau, A., G. Kruszewski, G. Lampl, L. Barrault, and M. Baroni (2018). What you can cram into a single $\backslash \$ \&$ !** vector: Probing sentence embeddings for linguistic properties. In Proceedings of ACL.

Das, A., S. Kottur, K. Gupta, A. Singh, D. Yadav, J. M. Moura, D. Parikh, and D. Batra (2017). Visual Dialog. In Proceedings of the IEEE Conference on Computer Vision and Pattern Recognition.

de Vries, H., F. Strub, S. Chandar, O. Pietquin, H. Larochelle, and A. C. Courville (2017). Guesswhat?! Visual object discovery through multi-modal dialogue. In Conference on Computer Vision and Pattern Recognition (CVPR).

Dobnik, S., M. Ghanimifard, and J. D. Kelleher (2018). Exploring the functional and geometric bias of spatial relations using neural language models. In Proceedings of the First International Workshop on Spatial Language Understanding (SpLu), pp. 1-11.

He, K., X. Zhang, S. Ren, and J. Sun (2016). Deep residual learning for image recognition. In Proceedings of the IEEE conference on computer vision and pattern recognition, pp. 770-778.

Johnson, J., B. Hariharan, L. van der Maaten, L. Fei-Fei, C. L. Zitnick, and R. Girshick (2017). Clevr: A diagnostic dataset for compositional language and elementary visual reasoning. In Proceedings of CVPR 2017.

Kazemzadeh, S., V. Ordonez, M. Matten, and T. L. Berg (2014). Referit game: Referring to objects in photographs of natural scenes. In EMNLP.

Kingma, D. P. and J. Ba (2014). Adam: A method for stochastic optimization. arXiv preprint arXiv:1412.6980.

Kriegeskorte, N., M. Mur, and P. A. Bandettini (2008). Representational similarity analysis-connecting the branches of systems neuroscience. Frontiers in systems neuroscience 2, 4.

Lin, T.-Y., M. Maire, S. Belongie, J. Hays, P. Perona, D. Ramanan, Dollar, P., and C. L. Zitnick (2014). Microsoft COCO: Common objects in context. In Proceedings of ECCV (European Conference on Computer Vision). 
Linzen, T., E. Dupoux, and Y. Goldberg (2016). Assessing the Ability of LSTMs to Learn SyntaxSensitive Dependencies. Transactions of the Association for Computational Linguistics 4, 521-535.

Madhysastha, P., J. Wang, and L. Specia (2018). Defoiling foiled image captions. In Conference of the North American Chapter of the Association for Computational Linguistics: Human Language Technologies, New Orleans, LA.

Mostafazadeh, N., I. Misra, J. Devlin, M. Mitchell, X. He, and L. Vanderwende (2016, August). Generating natural questions about an image. In Proceedings of the 54th Annual Meeting of the Association for Computational Linguistics (Volume 1: Long Papers), Berlin, Germany, pp. 1802-1813. Association for Computational Linguistics.

Patterson, K. and M. A. L. Ralph (2015). Neurobiology of Language, Chapter The Hub-and-Spoke Hypothesis of Semantic Memory. Elsevier.

Razavian, A. S., H. Azizpour, J. Sullivan, and S. Carlsson (2014). CNN features off-the-shelf: an astounding baseline for recognition. In Proceedings of the IEEE Conference on Computer Vision and Pattern Recognition Workshops, pp. 806-813.

Shekhar, R., S. Pezzelle, Y. Klimovich, A. Herbelot, M. Nabi, E. Sangineto, and R. Bernardi (2017). "foil it! find one mismatch between image and language caption". In Proceedings of the 55th Annual Meeting of the Association for Computational Linguistics (ACL) (Volume 1: Long Papers), pp. 255265.

Suhr, A., M. Lewis, J. Yeh, and Y. Artzi (2017, July). A corpus of natural language for visual reasoning. In Proceedings of the Annual Meeting of the Association for Computational Linguistics, Vancouver, Canada, pp. 217-223. Association for Computational Linguistics.

Wieting, J. and D. Kiela (2019). No training required: Exploring random encoders for sentence classification. In ICLR (accepted).

Yu, L., P. Poirson, S. Yang, A. C. Berg, and T. L. Berg (2016). Modeling context in referring expressions. In European Conference on Computer Vision, pp. 69-85. Springer.

Zhang, K. W. and S. R. Bowman (2018). Language modeling teaches you more syntax than translation does: Lessons learned through auxiliary task analysis. In Proceedings of the 2018 EMNLP Workshop BlackboxNLP: Analyzing and Interpreting Neural Networks for NLP, pp. 359-361. Association for Computational Linguistics. 